\title{
Co-designing Edutainment Applications with Kindergarten Teachers in an Online Setting
}

\author{
Adriana-Mihaela Guran, Grigoreta-Sofia Cojocar \\ Affiliation: Faculty of Mathematics and Computer Science, Babeş-Bolyai University, Cluj- \\ Napoca \\ Address: 1, M. Kogãlniceanu Street \\ E-mail: adriana.guran@ubbcluj.ro,grigoreta.cojocar@ubbcluj.ro
}

\begin{abstract}
The technological progress from the last decades has created the context for new changes in education. Integrating technology in the learning process for school age children is one of the challenges of this decade. Unfortunately, there is no focus on preschoolers that are born digital natives. In this paper, we address the problem of designing edutainment applications for preschoolers with the participation of educational experts, the kindergarten teachers. We describe how we have adapted the activities from the Participatory Design approach in the context of COVID19 pandemic, where social distancing was required, and we had to move all the design activities in an online setting. The results of our investigation show that participatory design is possible even in an online setting, but some limitations are determined by the lack of in-person interaction. At the same time, an advantage due to the flexibility and shared access to resources is underlined.
\end{abstract}

Keywords: participatory design, online, edutainment

DOI: 10.37789/ijusi.2021.14.1.2

\section{Introduction}

In the last years, more and more focus on the need for an upgrade in the education approaches has been raised. The recent progress of technology has opened new horizons for the future work context, and the trend of digitalization in every aspect of our life is increasingly evident each day. The requirement for digital citizens forces the need for developing digital skills in the young European Union citizens. The use of technology in the teaching process raises many debates from the perspective of replacing teachers by teacher-bots, based on the advances in Artificial Intelligence (Popenici, 2017; Chatterjee et al., 2020 ). Our research in developing edutainment applications for preschoolers has the main goal of introducing digital competences 
elements to young children by providing digital tools that support, but not replace, the kindergarten teachers' activities. We need to mention that developing edutainment applications for preschoolers is considered a support tool in regular activities. Statistics show that the role of kindergarten teachers is a complex one and it has a small probability of being replaced by computers (Benedikt, 2017).

As such, education experts have become the key for building fundamental digital skills in young children. For that, educators themselves need to integrate technology as a teaching/learning tool in their activities. But, to use technology in their regular teaching/learning activities, the digital resources must be appropriate for teachers' tasks and for children's age. Nowadays, different solutions of teaching using technology are used depending on the children's age. However, when the learners are preschoolers (aged 3-6 years in Romania) supplementary constraints on the design of edutainment applications occur. These constraints are determined by children's age and capabilities limitations. One of the proposed approaches in designing interactive applications that fit the educational requirements is to actively involve teachers in the design of these products. For edutainment applications for preschoolers, the kindergarten teachers are the most appropriate design partners, as they master the content children should learn, and, at the same time, they know their preferences and limitations. Children also play a role in the design of technology, but they cannot always be involved due to their early age and the educational role of the final products. Even more, the social distancing rules introduced by the COVID19 pandemic currently make it difficult to integrate the children in the design process.

In this paper, we describe the approach we have used for designing edutainment applications for preschoolers together with kindergarten teachers in an online setting. The paper begins with a brief introduction to the challenges in introducing technology into the teaching process of preschoolers, followed by a description of how edutainment can support this activity by including kindergarten teachers as co-designers. A case study on applying participatory design with kindergarten teachers in an online setting is described with a focus on the differences between applying in-person participatory design and participatory design in an online setting. The paper ends with a discussion of the obtained results, conclusions, and future research ideas. 


\section{Edutainment}

Introducing technology in the learning activities of preschoolers involves a mix between entertainment and educational content, to be acceptable. Edutainment applications provide both characteristics, being interactive applications that promote fun while providing educational content. The use of edutainment for preschoolers' activity seems an appropriate solution to help them build their fundamental digital skills and to support the development of an appropriate attitude towards technology. Children should be made aware of the fact that we are using technology not only for fun, but also to learn new things or to create new things. This way, by using entertainment, children get support in understanding technology as a tool for achieving goals by task performance.

The challenges in designing edutainment are determined by the two goals it must accomplish: being educative but also fun at the same time. Moreover, the final users of the edutainment applications are preschoolers (aged 3-6 years). The limitations determined by their age are the following:

- They cannot read.

- They cannot write.

- They cannot focus for long periods of times.

- They need rewards for accomplishing tasks.

- Their interaction actions are extremely limited.

So, to address both aspects, a deep understanding of what fun means for preschoolers and what are the goals and means of education at this age is needed. Therefore, the participation of kindergarten teachers in the design process is the appropriate method to ensure an appropriate solution. In the following we will present the process we have followed to support kindergarten teachers' participation through the design steps.

\section{Participatory Design}

Participatory Design (PD) is a design methodology in which the future users of a design participate as co-designers in the design process. In this methodology, participants (putative, potential or future) are invited to cooperate with designers, researchers and developers during an innovation process. Potentially, they participate during several stages of an innovation 
process: initial exploration, problem definition, and development. During the initial exploration and problem definition they help both define the problem and find ideas for solution, and during development, they help evaluate proposed solutions (Montemayor et. al, 2004). The key components that a codesign process should respect are:

- Intentionally involving target users in designing solutions;

- Postponing design decisions until after gathering feedback;

- Synthesizing feedback from target users into insights;

- Developing solutions based on feedback.

PD focuses on developing cooperative strategies for system design. It gives users a voice in the design process. The purpose of user participation is user empowerment and there is a distinction between functional empowerment and democratic empowerment. The functional empowerment means that the users should be able to carry out their work to their own satisfaction, and in an effective, efficient, and economical manner. Their participation in the design process supports reaching this goal. Democratic empowerment means that they should have the mandate to participate in decision-making in their workplace including the design and development of software and IT-based systems (Clement, 1994).

The users can play an informative, consultative, or participative role. As informants, users merely provide information about their work and might be the objects of some observations. In a consultative role, they are asked to comment on preset design solutions. In a participative role, they actively participate in the design process and have decision making power regarding the solution (Damodaran, 2006). Some of the techniques used in PD include interactive experimentation, modeling, testing, hands-on designing, and learning by doing. Low-tech prototypes can be drawn and created on blackboards, index cards, and paper. Through these techniques, adult system users communicate to system designers and developers the requirements for the system.

Mumford (Mumford, 1983) identifies two different forms of involvement: direct user participation and indirect user participation, where the user is represented by some intermediary. Direct and indirect participation are defined through the users' direct participation in the project (team) or their direct or indirect contact with project staff from the development organization. Previous research has shown that participatory design with 
children is possible, but the existing studies are describing experience with children aged 6 or above (Druin, 2002; Fails, 2009; Karlheins, 2010).

In this paper, we describe our participatory design approach used for the development of edutainment applications for Romanian preschool children in an online setting. The constraints introduced by the COVID19 pandemic required us to adapt our previous approach to a remote cooperation.

\subsection{Participatory Design with Preschoolers}

Before the COVID19 pandemic, to develop edutainment applications for preschoolers, a two-steps approach that involves both the kindergarten teachers and the young children have been applied (Guran\&Cojocar, 2019; Guran et al. 2019; Trischler et al., 2018). In the in-person participatory design approach children have been involved in every step of the design, except the alternative design step. For this step, due to potentially abstract design representations, the authors have replaced their participation with the participation of the kindergarten teachers. In the requirements step the design teams have performed field observation and interviews with the young children in their familiar environment, the kindergarten classroom. At the same time, interviews with kindergarten teachers have been organized to gather information about the educational requirements for the edutainment applications. Then, alternative designs have been generated. The alternative designs have taken the form of drawings, sketches, wireframes or even executable prototypes. If the alternative designs were not too abstract (like in the case of wireframes), children were asked to give their feedback on the proposed designs and even provide design suggestions related to the story that enveloped the learning content, the characters, and the objects that were presented. If the alternative designs were too abstract, the kindergarten teachers played the role of surrogate for the children, trying to imagine children's understanding and reactions. Afterward, the executable prototypes were evaluated with the children and kindergarten teachers' involvement. Although kindergarten teachers can predict children's behavior and reactions, some unexpected actions or misunderstandings have been revealed during playtesting sessions. So, in the in-person approach, children have been involved as informants, testers, and even co-designers in every step of the process. Kindergarten teachers have also been involved in every step of the design, by guiding the educational aspect in our edutainment applications and, sometimes, by trying to simulate children's behavior. The new global 
context restricts in-person interactions, moreover, it makes it impossible to interact in person with children during their regular activities in kindergartens. At present, when almost every activity has moved to a remote version, we have tried to adapt our participatory design approach to the new context, by using the technological progress introduced by communication platforms as a tool. As such, this paper tries to answer the following research questions:

- RQ1. Is it possible to apply online PD with kindergarten teachers to develop edutainment applications?

- $\quad \mathrm{RQ2}$. Are there any advantages of using an online PD approach?

- RQ3. Are there any disadvantages of using an online PD approach?

\section{Participatory Design in Online Setting - A Case Study}

The COVID19 pandemic and the adopted social distancing rules have restricted our access to the final users, and we needed to adapt our participatory design approach to the new context. So, we have considered that we could continue our work in developing edutainment applications for children by involving their educators, the kindergarten teachers as much as possible. We could not imagine a remote solution to involve the children in the design steps, as it would have been too unfamiliar for them to interact with new people. In these settings, interaction with the kindergarten teachers has taken place exclusively online. Even so, this communication constraint has brought some advantages, as we will present in the following.

In our study, four kindergarten teachers from a public kindergarten in our town, teaching children from the small group (aged 3-4 years aged) and the 3rd year students from the Faculty of Mathematics and Computer Science from our university were involved. The students have worked in teams during one semester to develop edutainment applications appropriate for 3-4 years old children by cooperating with the kindergarten teachers.

First, we have contacted one kindergarten teacher to present our intention to develop edutainment applications that integrate concepts established by the preschoolers' curriculum. We have described the motivation of our approach in terms of general directions in the European Union towards digitalization, the poor ranking of our country in terms of citizens' digital skills and the need to form a good fundamental basis for children' attitude towards technology. 
We have motivated our need for the participation of an educational expert that could guide the design process. The kindergarten teacher was enthusiastic about our approach and expressed her opinion on how the design should be addressed. The first important thing she mentioned was that different edutainment applications should be conceived for different age groups. The kindergarten teacher has also suggested the participation of other colleagues that are teaching children at the same level. In the selected kindergarten there are four classes of children at each level. She came with the idea of proposing the participation of all her colleagues in the design process. After all the colleagues have agreed to participate in our design initiative, the kindergarten teachers have organized brainstorming sessions where they have decided the domains from the curriculum that the edutainment applications should address, and they have produced an initial idea for the structure of the application. The kindergarten teachers came with the idea of edutainment simulating the habitual teaching activities that are usually split in two parts: one part for introducing the intended content followed by a practical part which consists of tasks that support the integration of the newly introduced knowledge.

The design and development of edutainment applications was performed by 3rd year students from the Faculty of Mathematics and Computer Science attending the Human-Computer Interaction optional course. The students have been split into 8 teams of 3-5 members. The kindergarten teachers have also been split into two teams of two people each, to work with four students' teams. The first meeting between the students and the kindergarten teacher took place online, using the Microsoft Teams platform. One kindergarten teacher has expressed their need for educational applications that can be used in both regular teaching activities as a support tool and online activities when children stay at home due to the pandemic. Students have addressed questions regarding the childrens' characteristics, their capacities and limitations, and have asked details about the subjects of the edutainment applications to be designed and developed. All kindergarten teachers have interacted with the students and provided details on their work with the preschoolers, on their usual activities and the constraints on working with such young children. Afterwards, each team of students received a problem statement (the subject) for the edutainment application. An example of a problem statement is shown in Table 1. An online meeting between each team of students and the team of two kindergarten teachers took place after the problem statement distribution. 
Table 1 - Initial problem statement example.

\section{DOMESTIC ANIMALS}

Age level: 3-4 years (small group)

INTEGRATED DOMAINS:

SCIENCE: ENVIRONMENTAL KNOWLEDGE (Information of interest about pets-food, benefits); MATHEMATICAL ACTIVITY (numbering in the limits 13 , spatial positions);

LANGUAGE AND COMMUNICATION DOMAIN (sentence composition, plural: one / many); THE HUMANS AND SOCIETY DOMAIN (ways of protecting domestic animals);

Thus during the requirements gathering step the kindergarten teachers have been directly involved as informants. Next, each students team has prepared three alternative designs for the edutainment applications that have been presented to the kindergarten teachers together with a user story. The design alternatives have taken the form of paper-based drawings or even executable prototypes. Examples of alternative designs are shown in Figure 1 and Figure 2. The teams presented the character that would guide the children in the interaction, the story that would incorporate the learning content and some ideas of tasks the children would perform to strengthen the newly acquired knowledge (as the ones with farmer Mitrut shown in Figure 3 and Figure 6). The following template was used: the character invites the children on a journey where they must help the character solve some tasks to gain a reward or to find the solution to a problem. In the shown example, children are invited to help farmer Mitrut answer some questions or solve memory games (as in Figure 5) to gain hay bales for his animals. 


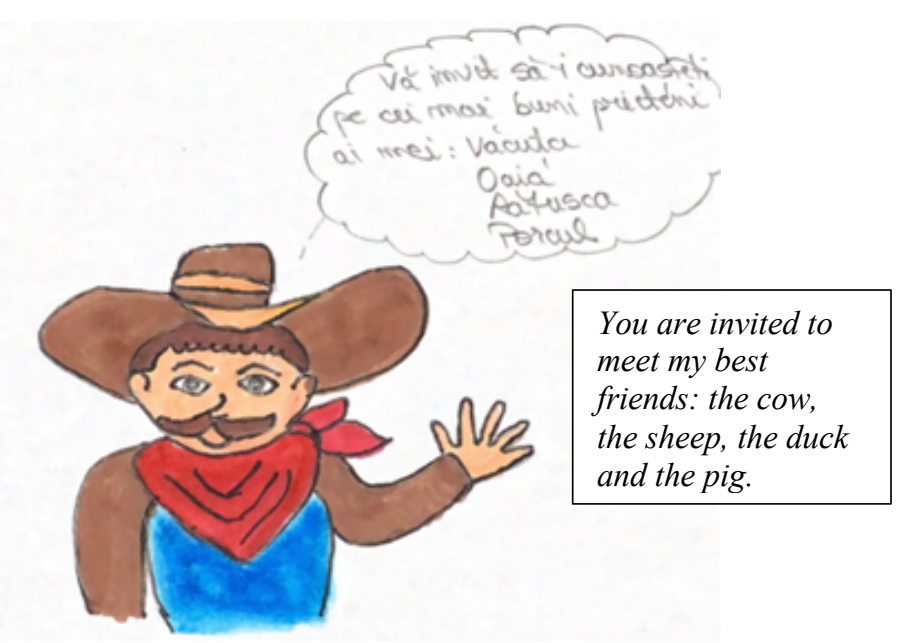

Figure 1. Alternative design for Domestic Animals

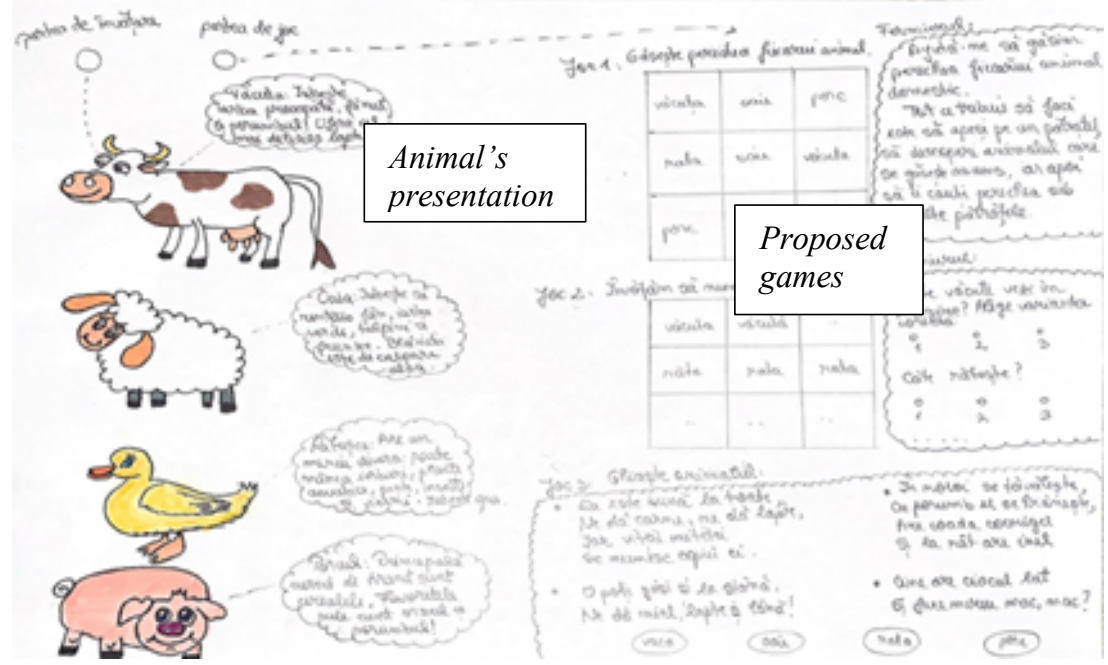

Figure 2. Alternative design for Domestic Animals edutainment/ content presentation part

In this step, the kindergarten teachers have taken the designer's role, and, starting from the ideas presented by the students, they suggested new story ideas to integrate the appropriate knowledge. Students and kindergarten teachers had multiple online brainstorming sessions where they agreed on what can be implemented and how. Afterwards, the student teams implemented the agreed ideas in executable prototypes. During this step, the 
collaboration between the students and the kindergarten teachers was both synchronous and asynchronous. Teachers have spent time on their own focusing on the story that could be developed to support the learning process and the proposed tasks/games, but also in brainstorming sessions with the students to identify possible implementations of the design ideas. The elaborated stories have been sent to the students as narrations that students needed to transpose in edutainment applications. There were difficulties in the design process when the problem of handling errors arose. Students' teams have proposed to let children go to the next question only if the children gave the right answer. Kindergarten teachers proposed to let the children try for at most 45 seconds and if the child didn't provide the right answer, the edutainment should automatically move to the next question. Some teams have also suggested providing clues to support children in solving the tasks, with penalties being applied to the final score of the child depending on the number of hints needed. The solution to design problems have always come to a consensus between students and the kindergarten teachers after exploring more possible options, and the time and resources constraints determined by the structure of the academic year. Kindergarten teachers have also provided feedback on the characters from the stories and on the sequence and complexity of tasks. Moreover, each application ends with rewards for the children which are differentiated based on the children's performance. For example, in the shown edutainment, the reward consists of hay bales that can be used to feed farmer's Mitrut animals (see Figure 6).

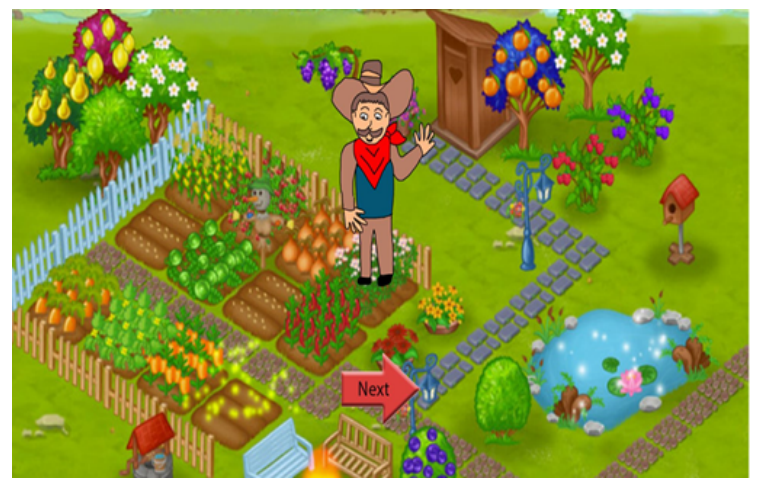

Figure 3. Introducing Mitruț farmer 


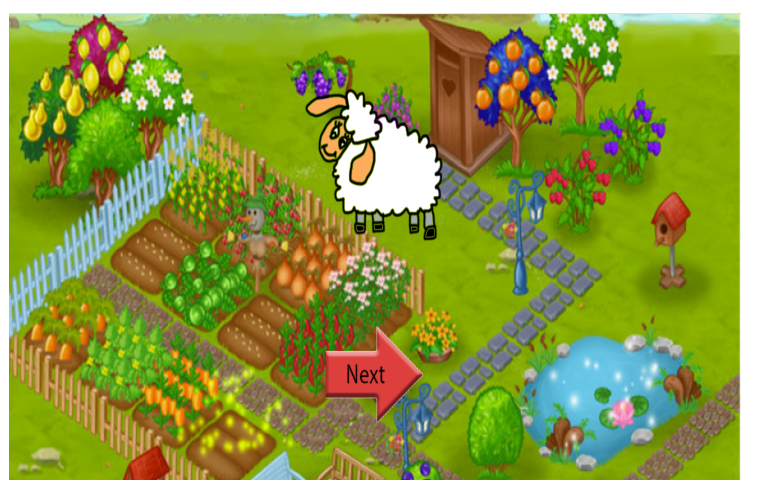

Figure 4. Content presentation window of Domestic Animals edutainment

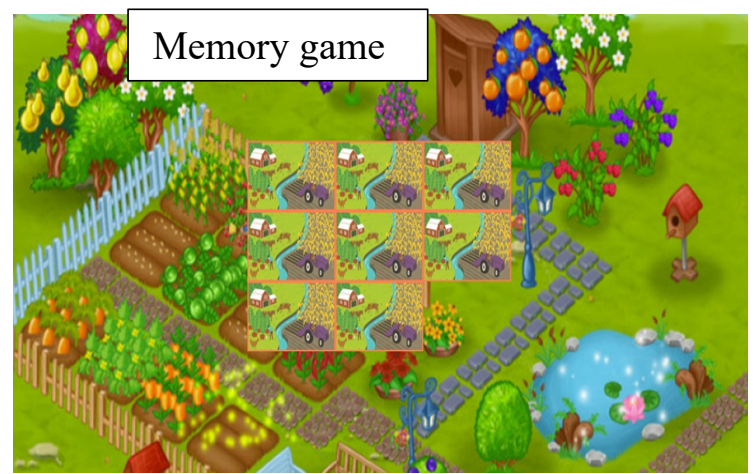

Figure 5. Memory game for Domestic Animals edutainment

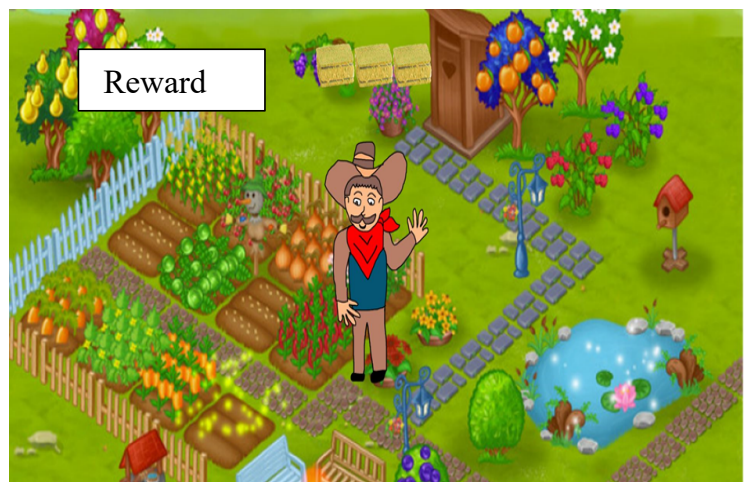

Figure 6. Final page with rewards (three hay bales)

After the prototype's development, students and kindergarten teachers met again in online meetings where the kindergarten teachers observed and tested 
the prototype. In this step kindergarten teachers have played the informants, testers and users' roles. Based on kindergarten teachers' feedback the students have implemented the required changes. In this step, kindergarten teachers have made observations on the sound synchronization, on the objects' movement on the screen for the task's objects, on the position of the navigational widgets on the screen (arrows to go to the next task, button to replay the audio content) and on the audio feedback given to the children, especially when they give a wrong answer. Online user testing sessions have been organized with young children using Zoom platform, with the applications running on students' computers and giving control to the children's devices. Children were always accompanied by at least one parent. The most significant aspect of these user testing sessions was children's attitude towards their experience of using the edutainment applications, which could not be assessed by interacting only with kindergarten teachers. In most cases children intended to play the entertainment applications again, even more than twice.

This way, the kindergarten teachers have been involved in each step of the design process, due to the opportunities given by the online communication platforms like Microsoft Teams and Zoom. In Table 2 is presented the synthesis of the kindergarten teachers' design participation, described by a role definition matrix (Barendregt et al., 2016), where $\mathrm{R}$ stands for Requirements, D stands for Design and E stands for Evaluation.

Table 2 - The role definition matrix for kindergarten teachers
\begin{tabular}{|l|l|l|l|}
\hline Activity in relation to designer & R & D & E \\
\hline Elaboration & $\mathrm{x}$ & $\mathrm{x}$ & \\
\hline Dialogue & $\mathrm{x}$ & $\mathrm{x}$ & $\mathrm{x}$ \\
\hline Feedback & & $\mathrm{x}$ & $\mathrm{x}$ \\
\hline Indirect & & & \\
\hline
\end{tabular}

\subsection{Comparison with in person participatory design}

In the previous preschoolers' edutainment design approaches used, participatory design with in-person interaction between all the stakeholders have been applied. When applying in-person participatory design the student teams and the kindergarten teachers participated in co-design activities by 
direct interaction, in scheduled meetings usually organized in the university classrooms. Any additional communication was performed only through emails. Very often, each students' team had one meeting with the kindergarten teachers in each step of the design. However, in an online setting, many student teams met multiple times with the kindergarten teachers, the online meetings allowing for a more flexible approach in scheduling them. Also, the platform used for online meetings allowed the students to record the meetings for future analysis.

An important disadvantage of the online setting is the lack of young children's involvement. Children could not be involved more in this process due to their limited access to technology, their lack of patience in the context of online teaching activities and the unfamiliar proposed tasks and unfamiliar people from the design teams.

\subsection{Discussion}

In the previous sections we have described our experience of using a remote (online) PD with only kindergarten teachers to design and develop edutainment applications for pre-schoolers. In the following, we will answer the research questions based on our experience.

\section{RQ1. Is it possible to apply online PD with kindergarten teachers to develop edutainment applications?}

Online participatory design with kindergarten teachers is possible. The existence of communications platforms like Microsoft Teams and Zoom makes it possible to have good and effective communication with the clients of the project. The options of sharing content (requirements lists, design sketches) and comment on them asynchronously, and the possibility of having synchronous meetings for brainstorming or clarifying design aspects helped the design teams. Recording of design meetings is another important feature of a communication platform that allows the design team members to review unclear aspects by replaying the discussions with the client. Also, the existence of a feature that permits to switch the control from one user to another allows clients to interact with a prototype without the need to install the application on their device.

\section{RQ2. Are there any advantages of using an online PD approach?}

The answer to this research question is clearly yes. The main advantage mentioned by the kindergarten teachers and design teams was the flexibility 
to schedule the meetings. In the offline settings, students and kindergarten teachers met only during the lecture classes when the kindergarten teacher was visiting the students, two or at most three times during the semester. During these lectures the teachers discussed with the design teams approximately two hours. The rest of the communication was performed using emails, and, sometimes, there were lengthy delays between the moment a question was raised by the design team and the receival of the answer, as kindergarten teachers did not check their emails regularly.

\section{RQ3. Are there any disadvantages of using an online PD approach?}

The answer to this research question is particularized for the members of the design teams and the kindergarten teachers. The members of the design teams felt that they needed to get to know the children and the kindergarten teachers in-person to feel more comfortable. Related to the design issues, the lack of in-person interaction with the final users influenced the design. The design teams' members needed to support their design decisions based only on what the kindergarten teachers have mentioned during the meetings. Some essential information that seemed obvious from kindergarten teachers' point of view was not known by the students (e.g., students considered that children can read statements or that they can perform drag and drop with the mouse). The kindergarten teachers considered that the design cycle was improved due to the existence of the communication platforms. This can be explained by the fact that in the past kindergarten teachers made the effort to visit the students in the university to get involved in the design process. The only disadvantage mentioned by the kindergarten teacher was the lack of children's involvement.

\section{Conclusions}

In this paper, we have described our first experience of applying participatory design without in-person interaction. In the previous edutainment design experiences, all the cooperation tasks have been performed by in-person meetings where open discussions and brainstorming sessions have been conducted to generate design ideas and implementation solutions. In this study we have replaced in-person meetings with online meetings, and we have discovered that online activities have their advantages, consisting in a more flexible approach in scheduling the meetings, the possibility of 
recording the meetings, and a more direct and frequent communication. Considering our first co-design experience using online platforms, we have decided that in the future, we will keep scheduling the meetings where feedback is needed as online meetings, due to their flexibility and easy access to each member of the design team (student or kindergarten teacher).

In the future, when the COVID19 pandemic will allow it, we intend to evaluate these edutainment applications with young children using an inperson approach, also, to obtain more realistic feedback from them.

\section{Acknowledgments}

We would like to thank the kindergarten teachers for being enthusiastic and open to collaboration, and to the students for their effort of conducting participatory design while learning new technologies that support the development of engaging edutainment.

\section{References}

Barendregt W., Bekker T., Börjesson P., Eriksson E., Torgersson O. 2016. The Role Definition Matrix: Creating a Shared Understanding of Children's Participation in the Design Process. Interaction Design and Children 10.1145/2930674.2935999.

Benedikt, F. K., Osborne, M.A. 2017. The Future of Employment: How Susceptible Are Jobs to Computerisation? Technological Forecasting and Social Change 114(C): 254-280

Bjerknes, G., and Bratteteig, T. 1995. User Participation and Democracy: A Discussion of Scandinavian Research on System Development, Scandinavian Journal of Information Systems (7:1), pp. 73-98.

Chatterjee S., Bhattacharjee K.K. 2020. Adoption of Artificial Intelligence in Higher Education: a Quantitative Analysis Using Structural Equation Modelling, Forthcoming in Education and Information Technologies (2020);

Clement, A. (1994) Computing at Work: Empowering Action By 'Low-level Users, Communications of the ACM (37:1), pp. 52-63

Damodaran, L. (2006) User Involvement in the Systems Design Process: A Practical Guide for Users, Behaviour and Information Technology (15:6), pp. 363-377, 2006.

Druin, A. 2002. The role of children in the design of new technology," Behaviour and Information Technology (BIT), vol. 21, no. 1, pp. 1-25, 2002.

Fails, J. A. .2009. Mobile Collaboration for Young Children: Reading and Creating Stories. College Park: University of Maryland, 2009.

Guran A-M, Cojocar G. S. 2019. Child-Centered Design of Edutainment Applications for Preschoolers. A two step method proposal, Workshop on Information and Communication Technologies, 27 th International Conference on Software 
Telecommunications and Computer Networks (SoftCOM 2019 conjoint event), http://softcom2019.fesb.unist.hr/, ISSN 1847-3598, p. 16-21, 2019.

Guran A-M, Cojocar G S, Moldovan A. 2019. Applying UCD for Designing Learning Experiences for Romanian Preschoolers. A case study., Proceedings of INTERACT 2019 17th IFIP TC.13 International Conference on Human-Computer Interaction, LNCS, D. Lamas et al. (Eds.): INTERACT 2019, ISBN/ISSN: 978-3-030-29389-5, 589-594, 2019

Guran, A. M. Cojocar, G. S., Moldovan, A. 2020. Designing edutainment software for digital skills nurturing of preschoolers: a method proposal. In Proceedings of the ACM/IEEE 42nd International Conference on Software Engineering: Software Engineering in Society (ICSE-SEIS '20). Association for Computing Machinery, New York, NY, USA, 63-70. DOI:https://doi.org/10.1145/3377815.3381383

Karlheinz K. 2010. Participatory Design Activities and Agile Software Development. IFIP WG 8.2/8.6 InternationalWorking Conference on Human Benefit through the Diffusion of Information Systems Design Science Research, Mar 2010, Perth, Australia. pp.303316, ff10.1007/978-3-642-12113-5_18ff. ffhal-01

Montemayor J., Druin, A., Chipman, G., Farber, A. and Guha, M. L .2004. Tools for children to create physical interactive story rooms, Computers in Entertainment (CIE), vol. 2, no. 1, pp. 12-35, 2004.

Mumford, E. 1983. Designing Human Systems for New Technology: The ETHICS Method, Manchester, UK: Manchester Business School060407, 1983

Popenici S.A.D., Kerr S. 2017. Exploring the impact of artificial intelligence on teaching and learning in higher education Res. Pract. Technol. Enhanc. Learn. (RPTEL), 12 (22) (2017), pp. 1-13

Trischler, Jakob; Pervan, Simon J.; Kelly, Stephen J.; Scott, Don R. 2018. The Value of Codesign. Journal of Service Research. 21: 75-100. doi:10.1177/1094670517714060. 